\title{
Secondary cytoreduction in platinum-sensitive recurrent ovarian cancer: are we missing something?
}

\author{
Claudia Marchetti ${ }^{1}$, Andrea Rosati ${ }^{1,2}$, Giovanni Scambia ${ }^{1,2}$, Anna Fagotti ${ }^{1,2}$ \\ ${ }^{1}$ Department of Woman, Child and Public Health, Fondazione Policlinico Universitario A. Gemelli IRCCS, Rome, Italy; ${ }^{2}$ Catholic University of the \\ Sacred Heart, Rome, Italy \\ Correspondence to: Anna Fagotti. Gynecologic Oncology Division, Fondazione Policlinico Universitario A. Gemelli, IRCCS, Università Cattolica del \\ Sacro Cuore, Rome, Italy. Email: anna.fagotti@unicatt.it. \\ Provenance: This is an invited article commissioned by the Executive Editor Dr. Zhi-De Hu (Department of Laboratory Medicine, the Affiliated \\ Hospital of Inner Mongolia Medical University, Hohhot, China). \\ Comment on: Gockley A, Melamed A, Cronin A, et al. Outcomes of secondary cytoreductive surgery for patients with platinum-sensitive recurrent \\ ovarian cancer. Am J Obstet Gynecol 2019;221:625.e1-625.e14.
}

Submitted Nov 09, 2019. Accepted for publication Dec 13, 2019.

doi: 10.21037/atm.2019.12.94

View this article at: http://dx.doi.org/10.21037/atm.2019.12.94

Despite initial maximal surgical effort and successful response to medical treatment, recurrence is a common event in high-grade serous ovarian cancer (HGSOC), with $75 \%$ of women experiencing relapse within 2 years from diagnosis (1). At relapse, patients with platinum sensitive recurrent ovarian cancer (PSROC) are frequently retreated with platinum-based doublets (2). Furthermore, maintenance therapy should be offered, either with bevacizumab, or with Parp-inhibitors (PARPi) (3-5), if not previously administrated.

While the benefits of secondary cytoreductive surgery (SCS) have been established for decades in retrospective studies (6), two recent RCTs have recently raised some concerns about the survival improvement suggested in previous series and meta-analyses (7-9). In both studies, randomization between surgery followed by chemotherapy or chemotherapy alone was performed. In the DESKTOP III trial PSROC patients with a positive Arbeitsgemeinschaft Gynäkologische Onkologie (AGO) score (Eastern Cooperative Oncology Group performance status 0, ascites $\leq 500 \mathrm{~mL}$, and optimal cytoreduction without residual tumor at initial surgery) were included (7). A 5.6-month increase in median progression-free survival (from 14.0 to 19.6 months $(\mathrm{P}<0.01)$ was found in those patients who underwent optimal SCS. Results on the primary endpoint of the study, overall survival, are still pending. The GOG213 study was designed to assess the impact of both antiangiogenic target treatment (with bevacizumab) and SCS on overall survival in PSROC. GOG213 showed that although SCS can be safely performed in these patients, it does not improve overall survival $(8,9)$.

In this unclear scenario, we read with great interest the paper form Gockley et al. (10), recently published on AJOG. This retrospective cohort study included 626 women from six National Cancer Institute referral centers, diagnosed with PSROC (2004-2011); among them, 146 (23\%) underwent SCS and 480 (77\%) received chemotherapy. They found that patients who underwent SCS were younger $(\mathrm{P}=0.001)$, with an earlier stage disease at diagnosis $(\mathrm{P}=0.002)$ and longer disease-free intervals $(\mathrm{P}<0.001)$, compared with those taking chemotherapy alone.

In the propensity score-matched group, including 244 patients, they also found a median overall survival of 54 months in patients who received SCS, compared with 33 months in those receiving chemotherapy $(\mathrm{P}<0.001)$. $70 \%$ of women achieved no residual or optimal secondary cytoreduction, and no significant differences in complication rates were found between patients receiving SCS versus those having chemotherapy only. According to the sensitivity analysis, the survival benefit related to SCS could be explained by a lower incidence of multiple sites of relapse, ascites or carcinomatosis among women undergoing SCS.

Overall, the Authors have to be congratulated for the great effort of analyzing one of the larger series ever 
published on this topic. Nonetheless, some issues should be considered. First of all, as the Authors state, no pre-surgical criteria were established, suggesting that observed survival difference is more related with disease's presentation, than with surgical or clinical factors. Furthermore, the use of defined pre-surgical selection criteria for SCS would have probably allowed to increase the $70 \%$ rate of no residual/ optimal cytoreduction, which is the only significant prognostic factor at time of SCS. We acknowledge this value is similar to previously reported studies (7-9), but lower than others $(11,12)$.

Interestingly, neither the correlation between SCS and BRCA mutation status, nor the impact of SCS in women receiving new emerging PARPi treatments have been analyzed in the study, probably due to the period of enrollment of patients (2004-2011). Similarly, these data are also missing in the 2 completed RCTs. Nonetheless, this information is now crucial to better understand ROC evolution and management.

With this regard, in a recent multicentric retrospective study, SCS has shown to be extremely effective for BRCA wild type PSROC women, whereas BRCA $1 / 2$ mutated patients' prognosis seem to be related more with molecular tumor characteristics than with tumor resectability (11). Nonetheless, in another retrospective study on a homogeneous population of PSROC BRCA mutated women who are supposed to receive Olaparib, survival is significantly increased when SCS is performed before PARPi administration with respect to PARPi alone. Indeed, as shown in the first line setting, tumor burden removal increases effectiveness of the drug, while decreasing the risks of resistance development (12).

Finally, as upfront management of OC is rapidly changing, it is rather straightforward that our next patients will be even more different from those presented in the current published literature on SCS in PSROC. In fact, in the forthcoming future, an increasing number of women are supposed to receive PARPi in the first-line setting, as maintenance therapy $(13,14)$. It is reasonable that first PSROC after PARPi treatment show different biological behavior, with unexpected metastatic sites and pattern of diffusion, which could preclude surgery. Results from currently ongoing randomized trials will probably clarify this issue (15).

In conclusion, according to the study of Gockley et al., SCS might be a valid therapeutic option in PSROC management. The study limitations related to the retrospective design and risk of selection bias can be overcome in the light of new recently delivered data on the benefit of SCS in both BRCA wild type women, who are the majority of the cases, and BRCA mutated patients who receive PARPi after platinum-based chemotherapy $(11,12)$. Nonetheless, as the landscape of treatment is changing, also our disease's knowledge should evolve and we should take the challenge to precisely define the role of SCS in the era of personalized and molecular medicine.

\section{Acknowledgments}

None.

\section{Footnote}

Conflicts of Interest: The authors have no conflicts of interest to declare.

Ethical Statement: The authors are accountable for all aspects of the work in ensuring that questions related to the accuracy or integrity of any part of the work are appropriately investigated and resolved.

\section{References}

1. Siegel RL, Miller KD, Jemal A. Cancer statistics, 2018. CA Cancer J Clin 2018;68:7-30.

2. Pisano C, Bruni GS, Facchini G, et al. Treatment of recurrent epithelial ovarian cancer. Ther Clin Risk Manag 2009;5:421-6.

3. Mirza MR, Monk BJ, Herrstedt J, et al. Niraparib maintenance therapy in platinum-sensitive, recurrent ovarian cancer. N Engl J Med 2016;375:2154-64.

4. Pujade-Lauraine E, Ledermann JA, Selle F, et al. Olaparib tablets as maintenance therapy in patients with platinumsensitive, relapsed ovarian cancer and a BRCA1/2 mutation (SOLO2/ENGOT-Ov21): a double-blind, randomised, placebo-controlled, phase 3 trial. Lancet Oncol 2017;18:1274-84.

5. Coleman RL, Oza AM, Lorusso D, et al. Rucaparib maintenance treatment for recurrent ovarian carcinoma after response to platinum therapy (ARIEL3): a randomised, double-blind, placebo-controlled, phase 3 trial. Lancet 2017;390:1949-61.

6. Lorusso D, Mancini M, Di Rocco R, et al. The role of secondary surgery in recurrent ovarian cancer. Int J Surg Oncol 2012;2012:613980.

7. Bois AD, Vergote I, Ferron G, et al. Randomized 
controlled phase III study evaluating the impact of secondary cytoreductive surgery in recurrent ovarian cancer: AGO DESKTOP III/ENGOT ov20. J Clin Oncol 2017;35:5501.

8. Coleman R, Enserro D, Spirtos N, et al. A phase III randomized controlled trial of secondary surgical cytoreduction (SSC) followed by platinum-based combination chemotherapy (PBC), with or without bevacizumab (B) in platinum-sensitive, recurrent ovarian cancer (PSOC): a NRG Oncology/Gynecologic Oncology Group (GOG) study. J Clin Oncol 2018;36:5501.

9. Coleman RL, Enserro D, Herzog TJ, et al. A phase III randomized controlled trial of secondary cytoreductive surgery (SCS) followed by platinum-based chemotherapy (PC) platinum-sensitive, recurrent ovarian cancer (PSOC)—surgical parameters. In: International Gynecologic Cancer Society. Kyoto, Japan 2018.

10. Gockley A, Melamed A, Cronin A, et al. Outcomes of secondary cytoreductive surgery for patients with platinum-sensitive recurrent ovarian cancer. Am J Obstet
Gynecol 2019;221:625.e1-625.e14.

11. Marchetti C, De Leo R, Musella A, et al. BRCA Mutation Status to Personalize Management of Recurrent Ovarian Cancer: A Multicenter Study. Ann Surg Oncol 2018;25:3701-8.

12. Marchetti C, Rosati A, Scaletta G, et al. Secondary cytoreductive surgery in platinum-sensitive recurrent ovarian cancer before olaparib maintenance: Still getting any benefit? A case-control study. Gynecol Oncol 2019;155:400-5.

13. Moore K, Colombo N, Scambia G, et al. Maintenance Olaparib in Patients with Newly Diagnosed Advanced Ovarian Cancer. N Engl J Med 2018;379:2495-505.

14. González-Martín A, Pothuri B, Vergote I, et al. Niraparib in Patients with Newly Diagnosed Advanced Ovarian Cancer. N Engl J Med 2019. [Epub ahead of print].

15. Pujade-Lauraine E, Colombo N, Glasspool R, et al. OReO/ENGOT Ov-38: A Phase IIIb trial of olaparib maintenance retreatment in patients with epithelial ovarian cancer. Ann Oncol 2017;28:v330-54.
Cite this article as: Marchetti C, Rosati A, Scambia G, Fagotti A. Secondary cytoreduction in platinum-sensitive recurrent ovarian cancer: are we missing something? Ann Transl Med 2019;7(Suppl 8):S372. doi: 10.21037/atm.2019.12.94 